Supporting Information for JA075037Z

\title{
Dehydration-induced water disordering in a synthetic potassium gallosilicate natrolite
}

\author{
Yongjae Lee, ${ }^{1, *}$ Sun Jin Kim, ${ }^{2}$ Ivor Bull, ${ }^{3}$ Aaron J. Celestian, ${ }^{4}$ \\ John B. Parise, ${ }^{5}$ Chi-Chang Kao, ${ }^{6}$ Thomas Vogt ${ }^{7}$ \\ E-mail) yongjaelee@yonsei.ac.kr
}

\footnotetext{
${ }^{1}$ Department of Earth System Sciences, Yonsei University, Seoul 120-749, Korea

${ }^{2}$ Nano-Materials Research Center, Korea Institute of Science and Technology, Seoul 136-791, Korea

${ }^{3}$ BASF CATALYSTS LLC, 101 Wood Avenue, P. O. Box 770, Iselin, New Jersey 08830

${ }^{4}$ Department of Geography and Geology, Western Kentucky University, Bowling Green, KY 42101

${ }^{5}$ Department of Geosciences, State University of New York, Stony Brook, NY 11794

${ }^{6}$ National Synchrotron Light Source, Brookhaven National Laboratory, Upton, NY 11973

${ }^{7}$ Department of Chemistry and Biochemistry, University of South Carolina, Columbia, SC 29208
} 
Supplementary Table 1. Final refined atomic coordinates for K-GaSi-NAT-ort as a function of temperature. ${ }^{\text {a }}$

\begin{tabular}{|c|c|c|c|c|c|c|c|c|c|c|}
\hline \multicolumn{2}{|l|}{ Temperature } & ambient & $50 \mathrm{~K}$ & $150 \mathrm{~K}$ & $250 \mathrm{~K}$ & $300 \mathrm{~K}$ & $350 \mathrm{~K}$ & $400 \mathrm{~K}$ & $450 \mathrm{~K}^{*}$ & $500 \mathrm{~K}^{*}$ \\
\hline \multicolumn{2}{|l|}{ space group } & $I 2_{1} 2_{1} 2_{1}$ & $I 2_{1} 2_{1} 2_{1}$ & $I 2_{1} 2_{1} 2_{1}$ & $I 2_{1} 2_{1} 2_{1}$ & $I 2_{1} 2_{1} 2_{1}$ & $\bar{I} 42 d$ & $\bar{I} 42 d$ & I4 $2 d$ & I4 $2 d$ \\
\hline \multicolumn{2}{|l|}{${ }_{\mathrm{w}} R_{\mathrm{p}}(\%), R_{\mathrm{p}}(\%), \chi 2$} & $3.1,2.1,102$ & $5.2,3.5,37$ & $5.1,3.5,32$ & $4.8,3.3,26$ & $4.6,3.2,22$ & $7.3,4.6,48$ & $6.8,4.2,36$ & $7.5,4.8,37$ & $7.4,4.5,58$ \\
\hline \multicolumn{2}{|l|}{ water molecules/u.c. } & $11.5(5)$ & $11.3(4)$ & $11.3(3)$ & $11.4(5)$ & $11.6(3)$ & $9.8(9)$ & $8.6(7)$ & 0 & 0 \\
\hline \multirow[t]{3}{*}{ cell length $(\AA)$} & $a$ & $13.7948(3)$ & $13.7298(4)$ & $13.7405(3)$ & $13.7589(3)$ & $13.7587(3)$ & $13.7026(3)$ & $13.6873(2)$ & $12.7372(4)$ & $12.7587(4)$ \\
\hline & $b$ & $13.7061(3)$ & $13.5815(4)$ & $13.5979(3)$ & $13.6300(3)$ & $13.6550(3)$ & & & & \\
\hline & $c$ & $6.5916(1)$ & $6.5589(2)$ & $6.5632(2)$ & $6.5721(2)$ & $6.5728(2)$ & $6.5600(2)$ & $6.5445(2)$ & $6.5630(4)$ & $6.5681(3)$ \\
\hline \multirow{4}{*}{$\begin{array}{l}4 c \\
4 a\end{array}$} & $x$ & 0.0000 & 0.0000 & 0.0000 & 0.0000 & 0.0000 & 0.0000 & 0.0000 & 0.0000 & 0.0000 \\
\hline & $y$ & 0.2500 & 0.2500 & 0.2500 & 0.2500 & 0.2500 & 0.0000 & 0.0000 & 0.0000 & 0.0000 \\
\hline & $z$ & $0.1258(11)$ & $0.1221(16)$ & $0.1221(15)$ & $0.1217(14)$ & $0.1207(13)$ & 0.0000 & 0.0000 & 0.0000 & 0.0000 \\
\hline & $U_{\text {iso }}$ & $0.006(1)$ & $0.005(1)$ & $0.003(1)$ & $0.006(1)$ & $0.006(1)$ & $0.007(1)$ & $0.020(1)$ & 0.020 & 0.020 \\
\hline \multirow[t]{3}{*}{$\mathrm{T}(21)$} & $x$ & $0.9699(2)$ & $0.9619(3)$ & $0.9622(3)$ & $0.9625(2)$ & $0.9629(2)$ & $-0.0321(2)$ & $-0.0315(2)$ & 0.9389 & 0.9394 \\
\hline & $y$ & $0.3831(2)$ & $0.3865(3)$ & $0.3867(3)$ & $0.3871(2)$ & $0.3875(2)$ & $0.1325(2)$ & $0.1331(2)$ & 0.1337 & 0.1337 \\
\hline & $z$ & $0.7413(2)$ & $0.7457(3)$ & $0.7459(3)$ & $0.7463(2)$ & $0.7467(2)$ & $0.6178(2)$ & $0.6184(2)$ & 0.6207 & 0.6207 \\
\hline \multirow[t]{3}{*}{$\mathrm{T}(22)$} & $x$ & $0.1323(2)$ & $0.1303(3)$ & $0.1299(2)$ & $0.1295(2)$ & $0.1290(2)$ & & & & \\
\hline & $y$ & $0.2802(2)$ & $0.2866(3)$ & $0.2862(2)$ & $0.2858(2)$ & $0.2853(2)$ & & & & \\
\hline & $z$ & $0.5033(2)$ & $0.5021(3)$ & $0.5017(2)$ & $0.5013(2)$ & $0.5008(2)$ & & & & \\
\hline \multirow[t]{4}{*}{$\mathrm{O}(11)$} & $x$ & $0.435(1)$ & $0.411(1)$ & $0.412(1)$ & $0.412(1)$ & $0.412(1)$ & $0.576(1)$ & $0.575(1)$ & 0.627 & 0.627 \\
\hline & $y$ & 0.000 & 0.000 & 0.000 & 0.000 & 0.000 & 0.250 & 0.250 & 0.250 & 0.250 \\
\hline & $z$ & 0.250 & 0.250 & 0.250 & 0.250 & 0.250 & 0.125 & 0.125 & 0.125 & 0.125 \\
\hline & $U_{\text {iso }}$ & $0.035(2)$ & $0.014(3)$ & $0.010(3)$ & $0.015(3)$ & $0.015(3)$ & $0.020(4)$ & $0.039(4)$ & 0.039 & 0.039 \\
\hline \multirow[t]{3}{*}{$\mathrm{O}(12)$} & $x$ & 0.250 & 0.250 & 0.250 & 0.250 & 0.250 & & & & \\
\hline & $y$ & $0.683(1)$ & $0.679(1)$ & $0.681(1)$ & $0.683(1)$ & $0.684(1)$ & & & & \\
\hline & $z$ & 0.000 & 0.000 & 0.000 & 0.000 & 0.000 & & & & \\
\hline \multirow[t]{3}{*}{$\mathrm{O}(21)$} & $x$ & $0.059(1)$ & $0.053(1)$ & $0.053(1)$ & $0.054(1)$ & $0.055(1)$ & $0.060(0)$ & $0.061(0)$ & 0.035 & 0.035 \\
\hline & $y$ & $0.375(1)$ & $0.380(1)$ & $0.379(1)$ & $0.378(1)$ & $0.379(1)$ & $0.130(0)$ & $0.127(0)$ & 0.144 & 0.144 \\
\hline & $z$ & $0.563(2)$ & $0.569(2)$ & $0.570(2)$ & $0.571(2)$ & $0.574(2)$ & $0.444(0)$ & $0.446(0)$ & 0.441 & 0.442 \\
\hline \multirow[t]{3}{*}{$\mathrm{O}(22)$} & $x$ & $0.126(1)$ & $0.124(1)$ & $0.124(1)$ & $0.124(1)$ & $0.124(1)$ & & & & \\
\hline & $y$ & $0.192(1)$ & $0.199(1)$ & $0.198(1)$ & $0.197(1)$ & $0.196(1)$ & & & & \\
\hline & $z$ & $0.685(2)$ & $0.687(2)$ & $0.685(2)$ & $0.683(2)$ & $0.681(2)$ & & & & \\
\hline \multirow[t]{3}{*}{$\mathrm{O}(31)$} & $x$ & $0.008(1)$ & $0.011(1)$ & $0.011(1)$ & $0.010(1)$ & $0.010(1)$ & $0.012(1)$ & $0.011(1)$ & -0.006 & -0.006 \\
\hline & $y$ & $0.355(1)$ & $0.354(1)$ & $0.354(1)$ & $0.354(1)$ & $0.354(1)$ & $0.104(1)$ & $0.104(1)$ & 0.109 & 0.109 \\
\hline & $z$ & $0.985(1)$ & $0.978(1)$ & $0.978(1)$ & $0.978(1)$ & $0.978(1)$ & $0.857(1)$ & $0.859(1)$ & 0.851 & 0.851 \\
\hline \multirow[t]{3}{*}{$\mathrm{O}(32)$} & $x$ & $0.104(1)$ & $0.102(1)$ & $0.102(1)$ & $0.102(1)$ & $0.103(1)$ & & & & \\
\hline & $y$ & $0.241(1)$ & $0.240(1)$ & $0.241(1)$ & $0.241(1)$ & $0.242(1)$ & & & & \\
\hline & $z$ & $0.264(1)$ & $0.267(1)$ & $0.267(1)$ & $0.266(1)$ & $0.264(1)$ & & & & \\
\hline \multirow[t]{5}{*}{$\mathrm{K} 1 \mathrm{~A}$} & $x$ & 0.250 & 0.250 & 0.250 & 0.250 & 0.250 & $-0.063(8)$ & $-0.065(6)$ & $-0.229(23)$ & $-0.169(8)$ \\
\hline & $y$ & $0.157(6)$ & $0.199(1)$ & $0.197(1)$ & $0.201(1)$ & $0.200(2)$ & 0.250 & 0.250 & 0.250 & 0.250 \\
\hline & $z$ & 0.000 & 0.000 & 0.000 & 0.000 & 0.000 & 0.125 & 0.125 & 0.125 & 0.125 \\
\hline & $P$ & $0.19(4)$ & $0.76(1)$ & $0.72(2)$ & $0.72(2)$ & $0.65(2)$ & $0.26(7)$ & $0.30(6)$ & $0.07(3)$ & $0.40(12)$ \\
\hline & $U_{\text {iso }}$ & $0.016(2)$ & $0.022(3)$ & $0.023(3)$ & $0.036(3)$ & $0.044(3)$ & $0.056(8)$ & $0.047(7)$ & $0.042(9)$ & $0.041(9)$ \\
\hline \multirow[t]{4}{*}{ K1B } & $x$ & 0.250 & 0.250 & 0.250 & 0.250 & 0.250 & $-0.008(6)$ & $-0.009(6)$ & $-0.147(3)$ & $-0.118(15)$ \\
\hline & $y$ & $0.210(3)$ & $0.286(4)$ & $0.278(4)$ & $0.276(4)$ & $0.271(4)$ & 0.250 & 0.250 & 0.250 & 0.250 \\
\hline & $z$ & 0.000 & 0.000 & 0.000 & 0.000 & 0.000 & 0.125 & 0.125 & 0.125 & 0.125 \\
\hline & $P$ & $0.59(3)$ & $0.24(1)$ & $0.28(2)$ & $0.28(2)$ & $0.35(2)$ & $0.48(6)$ & $0.41(5)$ & $0.61(3)$ & $0.31(10)$ \\
\hline \multirow[t]{4}{*}{$\mathrm{K} 1 \mathrm{C}$} & $x$ & 0.250 & & & & & & & & \\
\hline & $y$ & $0.279(4)$ & & & & & & & & \\
\hline & $z$ & 0.000 & & & & & & & & \\
\hline & $P$ & $0.24(2)$ & & & & & & & & \\
\hline
\end{tabular}




\begin{tabular}{|c|c|c|c|c|c|c|c|c|c|c|c|}
\hline \multirow[t]{4}{*}{$\mathrm{K} 2 \mathrm{~A}$} & \multirow[b]{2}{*}{$4 a$} & $x$ & $0.063(2)$ & $0.039(1)$ & $0.042(1)$ & $.0420(10)$ & $0.041(1)$ & $0.074(4)$ & $0.075(4)$ & $-0.035(4)$ & $-0.027(8)$ \\
\hline & & $y$ & 0.000 & 0.000 & 0.000 & 0.000 & 0.000 & 0.250 & 0.250 & 0.250 & 0.250 \\
\hline & \multirow[t]{2}{*}{$8 d$} & $z$ & 0.250 & 0.250 & 0.250 & 0.250 & 0.250 & 0.125 & 0.125 & 0.125 & 0.125 \\
\hline & & $P$ & $0.44(3)$ & $0.86(1)$ & $0.84(1)$ & $0.80(1)$ & $0.76(1)$ & $0.30(2)$ & $0.30(2)$ & $0.24(1)$ & $0.21(3)$ \\
\hline \multirow[t]{4}{*}{ K2B } & \multirow{4}{*}{$4 a$} & $x$ & $0.011(3)$ & $-0.099(5)$ & $-0.084(4)$ & $-0.078(3)$ & $-0.072(3)$ & & & & \\
\hline & & $y$ & 0.000 & 0.000 & 0.000 & 0.000 & 0.000 & & & & \\
\hline & & $z$ & 0.250 & 0.250 & 0.250 & 0.250 & 0.250 & & & & \\
\hline & & $P$ & $0.39(3)$ & $0.14(1)$ & $0.16(1)$ & $0.20(1)$ & $0.24(1)$ & & & & \\
\hline \multirow[t]{4}{*}{$\mathrm{K} 2 \mathrm{C}$} & \multirow{4}{*}{$4 a$} & $x$ & $-0.068(3)$ & & & & & & & & \\
\hline & & $y$ & 0.000 & & & & & & & & \\
\hline & & $z$ & 0.250 & & & & & & & & \\
\hline & & $P$ & $0.19(1)$ & & & & & & & & \\
\hline \multirow[t]{5}{*}{ OW1 } & \multirow{5}{*}{$\begin{array}{l}4 b \\
8 d\end{array}$} & $x$ & 0.250 & 0.250 & 0.250 & 0.250 & 0.250 & $0.215(9)$ & $0.199(8)$ & & \\
\hline & & $y$ & $-0.007(2)$ & $-0.019(2)$ & $-0.019(2)$ & $-0.026(2)$ & $-0.034(2)$ & 0.250 & 0.250 & & \\
\hline & & $z$ & 0.000 & 0.000 & 0.000 & 0.000 & 0.000 & 0.125 & 0.125 & & \\
\hline & & $P$ & 1.000 & 1.000 & 1.000 & 1.000 & 1.000 & $0.31(5)$ & $0.29(3)$ & & \\
\hline & & $U_{i s o}$ & $0.122(9)$ & $0.022(3)$ & $0.023(3)$ & $0.036(3)$ & $0.044(3)$ & $0.056(8)$ & $0.047(7)$ & & \\
\hline \multirow[t]{4}{*}{ OW2 } & \multirow{4}{*}{$\begin{array}{l}8 d \\
8 d\end{array}$} & $x$ & $0.219(2)$ & $0.221(3)$ & $0.228(3)$ & $0.220(2)$ & $0.219(3)$ & $0.298(5)$ & $0.296(5)$ & & \\
\hline & & $y$ & $0.515(4)$ & $0.502(4)$ & $0.499(4)$ & $0.509(4)$ & $0.504(4)$ & 0.250 & 0.250 & & \\
\hline & & $z$ & $0.659(4)$ & $0.613(5)$ & $0.602(4)$ & $0.611(4)$ & $0.613(4)$ & 0.125 & 0.125 & & \\
\hline & & $P$ & $0.60(3)$ & $0.45(2)$ & $0.44(2)$ & $0.51(3)$ & $0.49(2)$ & $0.50(5)$ & $0.40(4)$ & & \\
\hline \multirow[t]{4}{*}{ OW3 } & \multirow{4}{*}{$\begin{array}{l}8 d \\
8 d\end{array}$} & $x$ & $0.210(4)$ & $0.214(3)$ & $0.210(3)$ & $0.213(3)$ & $0.217(3)$ & $-0.214(4)$ & $-0.231(4)$ & & \\
\hline & & $y$ & $0.396(4)$ & $0.407(3)$ & $0.406(3)$ & $0.401(4)$ & $0.402(3)$ & 0.250 & 0.250 & & \\
\hline & & $z$ & $-0.053(9)$ & $-0.066(6)$ & $-0.073(6)$ & $-0.076(6)$ & $-0.088(6)$ & 0.125 & 0.125 & & \\
\hline & & $P$ & $0.34(3)$ & $0.46(3)$ & $0.47(2)$ & $0.42(3)$ & $0.46(2)$ & $0.42(2)$ & $0.38(2)$ & & \\
\hline
\end{tabular}

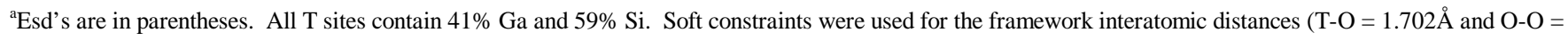
2.780 $)$. Restraints were used to set the isotropic displacement factors, $U_{i s o}\left(\AA^{2}\right)$, equal for the same atomic species (in ambient temperature model) or for the framework and nonframework species (in variable temperature models). The sum of the site occupancies for the K1 and K2 series was constrained to be unity in the $50 \mathrm{~K}$ to $300 \mathrm{~K}$ models. Upper and lower site symbols for $I 2_{1} 2_{1} 2_{1}$ and $\boldsymbol{I} 42 d$ phases, respectively. $*$ DLS optimized framework models fixed during refinement. 
Selected interatomic distances $(\AA)$ and angles $\left(^{\circ}\right)$ for K-GaSi-NAT-ort as a function of temperature. ${ }^{a}$






\begin{tabular}{|c|c|c|c|c|c|c|c|c|c|}
\hline $\mathrm{K} 2 \mathrm{~B}-\mathrm{K} 2 \mathrm{C}^{\mathrm{b}}$ & $1.08(4)$ & & & & & & & & \\
\hline OW1-O(11) & $3.036(12) \times 2$ & $2.77(1) \times 2$ & $2.77(1) \times 2$ & $2.79(1) \times 2$ & $2.81(1) \times 2$ & $2.93(2) \times 2$ & $2.98(3) \times 2$ & & \\
\hline OW1-K1A & $2.24(8)$ & $2.96(3)$ & 2.94(3) & $3.09(3)$ & $3.19(4)$ & $3.07(9) \times 2$ & $3.10(7) \times 2$ & & \\
\hline OW1-K1B & $2.97(4)$ & & & & & $3.06(14)$ & $2.85(12)$ & & \\
\hline OW1-K2A & $3.068(26) \times 2$ & $3.34(1) \times 2$ & $3.31(1) \times 2$ & $3.32(1) \times 2$ & $3.35(1) \times 2$ & & & & \\
\hline OW2-O(12) & $2.94(5)$ & $2.59(5)$ & $2.57(5)$ & $2.76(4)$ & $2.71(4)$ & $2.80(5) \times 2$ & $2.83(5) \times 2$ & & \\
\hline OW2-O(21) & $2.99(4)$ & $2.85(5)$ & $2.91(4)$ & $2.92(4)$ & $2.84(4)$ & & & & \\
\hline OW2-K1A & & & & & & $\begin{array}{l}3.11(10) \times \\
2\end{array}$ & $3.09(7) \times 2$ & & \\
\hline OW2-K1B & & $3.00(8)$ & $3.12(7)$ & $3.04(7)$ & $3.19(7)$ & & & & \\
\hline $\mathrm{OW} 2-\mathrm{K} 2 \mathrm{C}$ & $3.01(5)$ & & & & & & & & \\
\hline OW3-O(11) & & & & & & $2.88(5)$ & $2.65(5)$ & & \\
\hline OW3-O(12) & $3.19(6)$ & $3.12(4)$ & $3.09(4)$ & $3.05(4)$ & $2.99(4)$ & & & & \\
\hline OW3-O(21) & $3.29(5)$ & $3.28(4)$ & $3.20(4)$ & $3.21(4)$ & $3.16(4)$ & & & & \\
\hline OW3-O(31) & $2.86(6)$ & $2.89(5)$ & $2.85(4)$ & $2.89(5)$ & $2.95(5)$ & & & & \\
\hline OW3-K1A & & $2.90(4)$ & $2.93(4)$ & $2.82(5)$ & $2.86(5)$ & & & & \\
\hline OW3-K1B & $2.64(7)$ & $1.77(6)$ & $1.88(6)$ & $1.84(7)$ & $1.94(6)$ & $2.82(10)$ & $3.04(10)$ & & \\
\hline OW3-K2A & & & & & & $2.96(4) \times 2$ & $2.91(4) \times 2$ & & \\
\hline OW3-K2B & & $2.89(5)$ & $3.02(5)$ & $3.15(5)$ & $3.27(5)$ & & & & \\
\hline
\end{tabular}

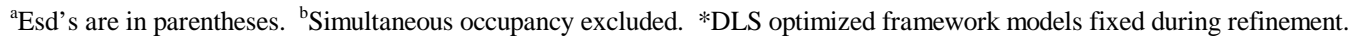


Supplementary Figure 1.

wt. loss near room temperature.

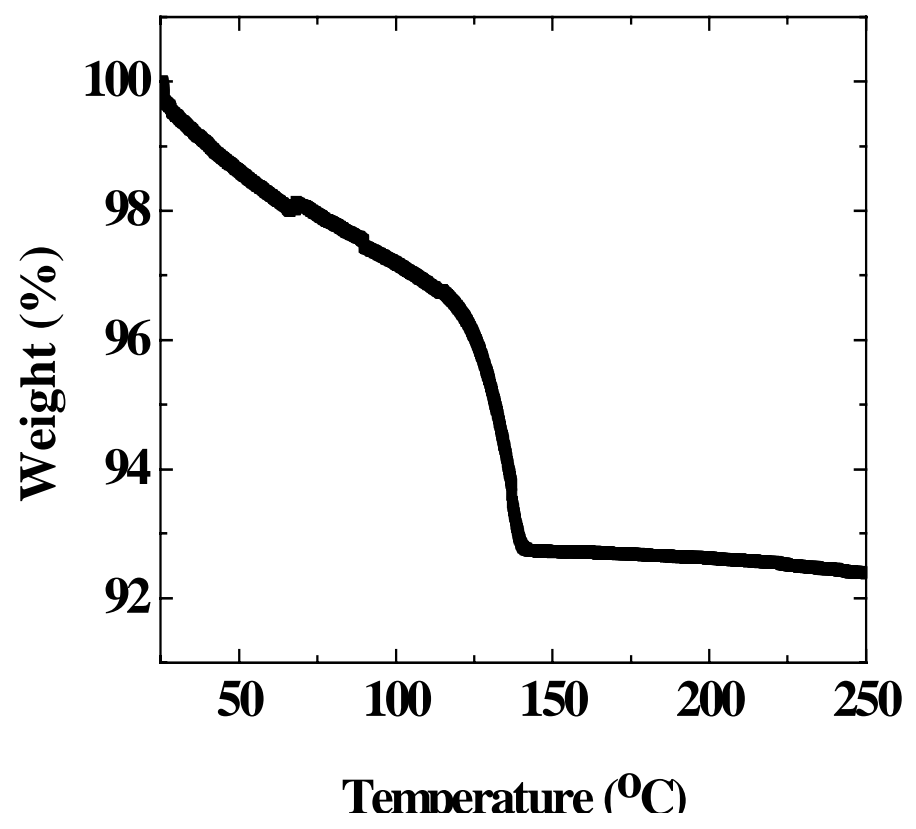



using synchrotron X-ray powder diffraction data. Points shown as + represent observed data. The continuous lines through the sets of points are the calculated profiles from the structure refinements summarized in Supplementary Tables 1-2. Fixed points were used to model the structured backgrounds. The sets of tic marks below the data indicate the positions of the allowed reflections. The lower curves represents the differences between observed and calculated profiles $\left(I_{\mathrm{obs}}-\right.$ $I_{\text {calc }}$ ) plotted on the same scale as the observed data.
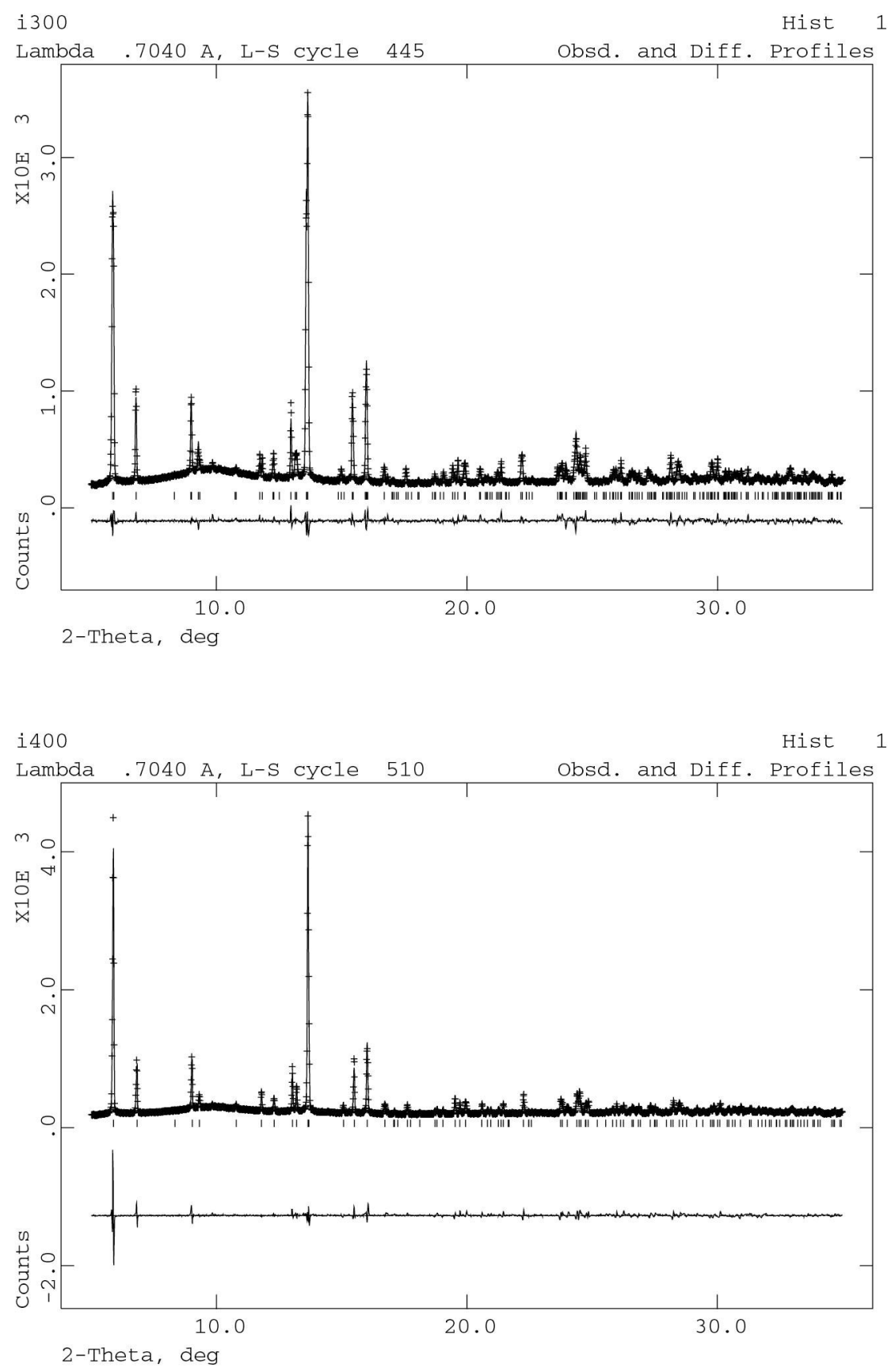